\title{
Taking the Risk Out of Risk Assessment
}

\section{Originating Technology/ NASA Contribution}

$\mathrm{T}$ The ability to understand risks and have the right strategies in place when risky events occur is essential in the workplace. More and more organizations are being confronted with concerns over how to measure their risks or what kind of risks they can take when certain events transpire that could have a negative impact.

NASA is one organization that faces these challenges on a daily basis, as effective risk management is critical to the success of its missions-especially the Space Shuttle missions.

On July 29, 1996, former NASA Administrator Daniel Goldin charged NASA's Office of Safety and Mission Assurance with developing a probabilistic risk assessment (PRA) tool to support decisions on the funding of Space Shuttle upgrades. When issuing the directive, Goldin said, "Since I came to NASA [in 1992], we've spent billions of dollars on Shuttle upgrades without knowing how much they improve safety. I want a tool to help base upgrade decisions on risk." Work on the PRA tool began immediately.

The resulting prototype, the Quantitative Risk Assessment System (QRAS) Version 1.0, was jointly developed by NASA's Marshall Space Flight Center, its Office of Safety and Mission Assurance, and researchers at the University of Maryland. QRAS software automatically expands the reliability logic models of systems to evaluate the probability of highly detrimental outcomes occurring in complex systems that are subject to potential accident scenarios.

Even in its earliest forms, QRAS was used to begin PRA modeling of the Space Shuttle. In parallel, the development of QRAS continued, with the goal of making it a world-class tool, one that was especially suited to NASA's unique needs. From the beginning, an important conceptual goal in the development of QRAS was for it to help bridge the gap between the professional risk analyst and the design engineer. In the past, only the professional risk analyst could perform, modify, use, and perhaps even adequately understand PRA. NASA wanted to change this by developing a PRA tool that would be friendlier, more understandable, and more useful to the broader engineering community. This concept ultimately led to the look, feel, and functionality that QRAS has today.

\section{Partnership}

In July 2003, Item Software (USA) Inc., of Anaheim, California, received an exclusive license for the QRAS software. The company is a leader in providing software solutions and services for reliability, availability, maintainability, safety, quality assurance, and risk assessment to government and commercial customers in aerospace and

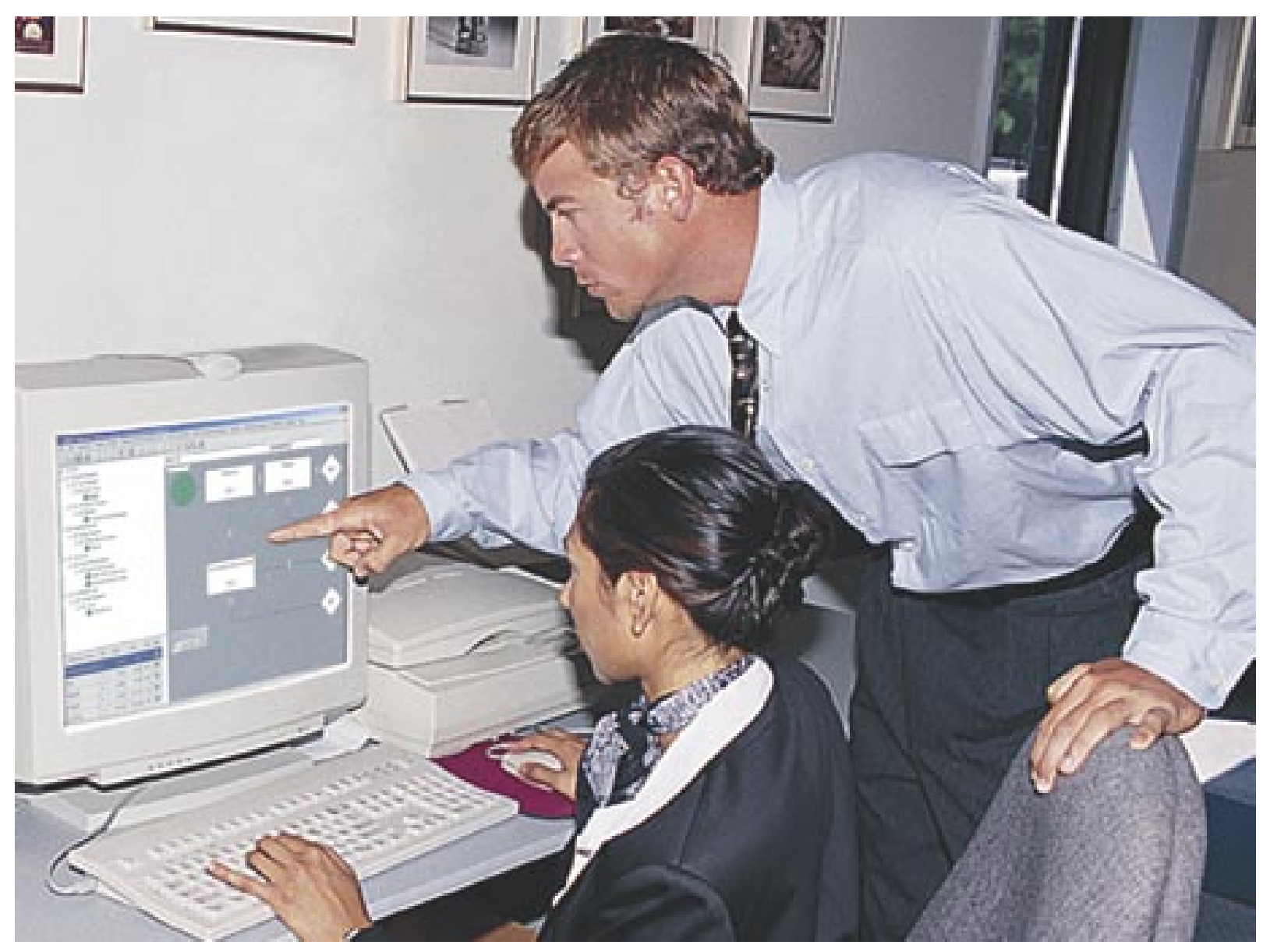

The Quantitative Risk Assessment System (QRAS) is a comprehensive tool for conducting probabilistic risk assessment. 
other industries. It built on the already-state-of-the-art features of QRAS, preparing the software for commercial sale as part of its suite of software and associated services. As part of the commercialization and distribution of QRAS, Item Software also supplies support and training.

The license provides the basis for a potential partnership between NASA and Item Software for the future extension and deployment of QRAS and related software, as may be needed for NASA purposes.

\section{Product Outcome}

Item Software developed the first commercialized version of QRAS and released it in January 2005. The new, enhanced QRAS 1.80 provides a cohesive and simple-yetpowerful platform for system risk assessment, through a large range of failure probability characterizations for engineering and scenario-driven applications.

The software builds a risk model of a system or a scenario for which risk of failure is being assessed, then analyzes the associated risk to the risk model. It can then be used to perform sensitivity analysis of the risk model by altering fundamental components and quantification models. During this stage, a fixed baseline is constructed and stored. This baseline contains the solutions for the lowest level scenarios, preserved in an event tree structure. The analysis, at any level of the hierarchy, aggregates these baseline results for risk quantitative computation as well as ranking of a particular risk.

The commercial version introduces substantial enhancements and includes many new features not seen in the original version. For example, it contains fault tree analysis, a deductive procedure for determining the various combinations of hardware and software failures, plus human errors that could result in the occurrence of specified undesired events. Fault tree analysis is one of the most widely used methods in system reliability analysis.

A new fault tree editor with extensive editing and user-friendly features was also added to the commercial version to allow users to effortlessly create, review, and analyze multiple fault trees simultaneously. The fault trees created with the fault tree editor can be attached to the event sequence diagram (ESD) in QRAS projects. An ESD is a visual representation of a set of possible outcome scenarios originating from an event. Each scenario in an ESD consists of a unique sequence of pivotal events, and eventually leads to an end-state that designates the severity of the outcome of a particular scenario.

QRAS assesses risk at the failure mode, subsystem, and element (i.e., a group of subsystems) levels, based upon user-supplied quantification of failure models, event sequence system decompositions, and system operating time. It provides users with structured guidance so managers, engineers, and even individuals who may not be experienced in the field of risk assessment can use it.

Overall, the QRAS software's unique, patented PRA capabilities assist risk analysts in modeling deviations from a system's nominal functions, the timing and likelihood

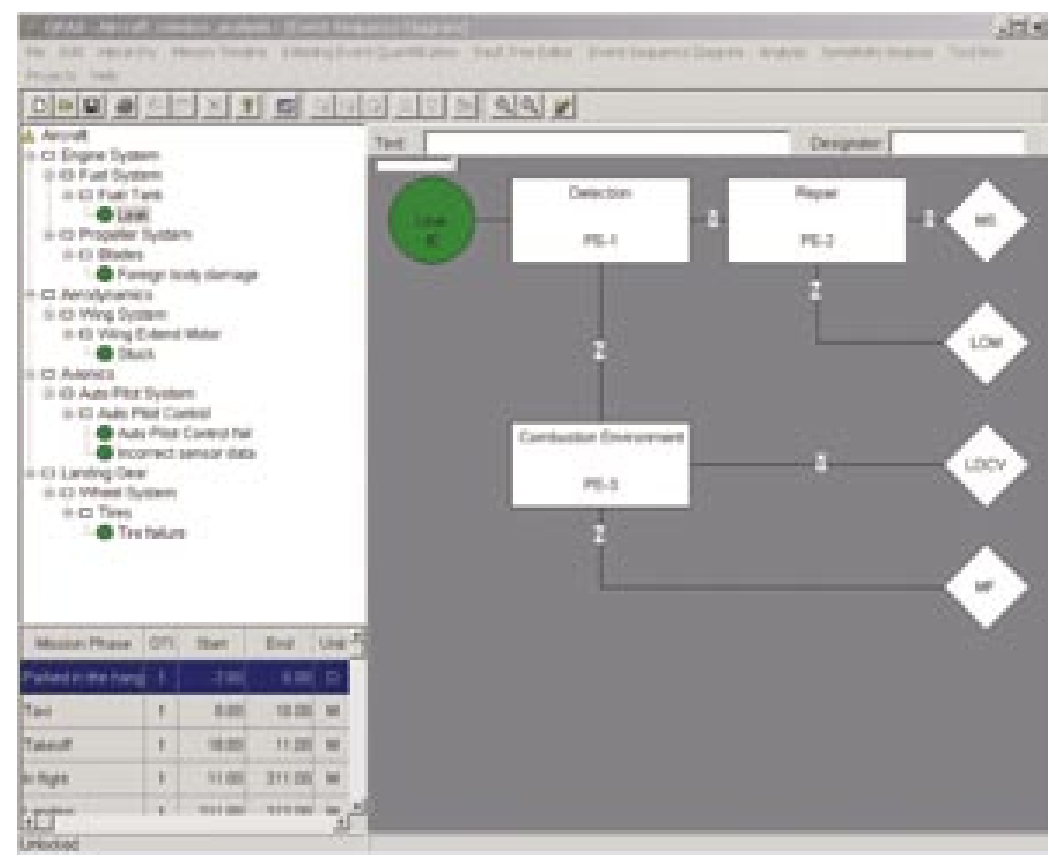

of such deviations, potential consequences, and scenarios leading from initial deviations to such consequences.

The U.S. Department of Homeland Security, The Boeing Company, Booz Allen Hamilton, Inc., ITT Industries, Inc., the European Space Agency, and the China National Space Administration are just some of the entities evaluating QRAS for their risk assessment needs.

Those currently employing it-other than NASAinclude the Canadian Space Agency, AES Corporation, and Harvard Medical School. At Harvard Medical School's major teaching hospital, Beth Israel Deaconess Medical School Center, QRAS is being utilized to determine the health care risk associated with general surgical processes and surgical devise use, pharmaceutical ordering, transfusion services, and organ procurement and transplantation.

Other examples of application include determining the probability of airplane crashes arising from factors such as engine failure, avionics failure, or human-failure at the air control tower; and train collisions caused by failures in train-signaling systems.

QRAS provides a full graphical user interface, including fault tree editors and event sequence diagrams, plus analysis screens that provide results in tabular and graphical formats. The screen to the left portrays System Hierarchy Mission Phase and event sequence diagrams that are used to determine the consequence of an initiating event and the expected frequency of each consequence. For example, a leak in an aircraft fuel system could result in the following user-defined end states: Mission Success (MS) if the leak is repaired, or Loss of Mission (LOM), Loss of Crew and Vehicle (LOCV), or Mission Failure (MF). 